Rev Chil Salud Pública 2009; Vol 13 (2): 90-99

ARTÍCULOS ORIGINALES

\title{
Factores asociados con el auto-reporte de maltrato en adultos mayores de México
}

\author{
MA. GUADALUPE RUELAS GONZÁLEZ ${ }^{(1)}$ y V. NELLY SALGADO DE ZINDER ${ }^{(1)}$
}

\section{RESUMEN}

Objetivo: Identificar algunos factores sociodemográficos, de salud fisica y mental, apoyo social $y$ de utilización de servicios de salud asociados con el auto-reporte de maltrato en una muestra de hombres y mujeres adultos mayores viviendo en colonias urbano-marginales de cuatro municipios de México. Método: Se llevó a cabo un estudio transversal entre junio del 2004 y agosto del 2005 con una muestra propositiva de 799 adultos mayores que residian en colonias urbanas, identificadas como de "muy alta" y "alta marginación" en los municipios de Cuernavaca, Chilpancingo, Guadalajara y Culiacán. Un cuestionario dirigido a los adultos mayores fue utilizado para explorar tanto la variable dependiente (auto-reporte de maltrato) como las variables asociadas: características sociodemográficas, estado de salud física y mental, apoyo social y familiar y utilización de servicios de salud. Para el análisis estadístico se emplearon pruebas no paramétricas así como un modelo multivariado de regresión logística. Resultados: En esta muestra de ancianos, el 23\% de las mujeres y el 9\% de los hombres reportaron haber sido maltratados como consecuencia de problemas familiares. Los resultados sugieren que los factores que se relacionan con el maltrato en los adultos mayores incluidos en este estudio son: sexo femenino $(R M=3.73 ; I C=1.76-4.24)$, edad de 60 a 69 años $(R M=1.61 ; I C=1.06-2.42)$, ser divorciado o separado $(R M=2.28 ; I C=1.32-3.96)$, padecer un mayor número de enfermedades $(R M=1.07 ; I C=1.01-1.17)$, presentar lesiones evidentes a la observación $(R M=2.89 ; I C=1.01-3.58)$, presentar sintomatología depresiva $(R M=1.68 ; I C=1.04$ 2.72), y no contar con apoyo familiar adecuado (RM=2.91;IC=1.86-4.55). Conclusión: Los sistemas de salud enfrentan un reto importante en el maltrato del adulto mayor debido, por un lado, al rápido aumento de este grupo poblacional y, por otro, a las carencias de recursos materiales y de recursos humanos especializados. El maltrato hacia los ancianos puede verse exacerbado por el aumento de la violencia social y los cambios en la estructura familiar y roles de género tradicionales. Enfrentar adecuadamente este problema requiere de una atención integral a las necesidades de los adultos mayores, con la participación de todos los sectores de la sociedad, profundizando en su conocimiento, sensibilizando a la sociedad y desarrollando estructuras que permitan la prestación oportuna y eficiente de servicios de salud.

Palabras clave: maltrato adultos mayores, poblaciones vulnerables, Mexico.

\section{ABSTRACT}

\section{FACTORS ASSOCIATED WITH SELF-REPORTING OF ABUSE IN SENIORS CITIZENS IN} MEXICO

Objective: Identify some sociodemographic factors, in physical and mental health, social support and utilization of health services, associated with the self-reporting of abuse in a sample of male and female senior citizens living in urban, marginalized settlements in four municipalities

(1) Instituto Nacional de Salud Pública. Avenida Universidad 655. Colonia Santa Maria Ahuacatitlán. Cuernavaca. Mor. CP 62508. México.nsnyder@insp.mx. 
of Mexico. Method: A cross sectional study was carried out between June of 2004 and August of 2005, with a purposive sample of 799 seniors who live in urban settlements identified as having "very high" or "high marginalization," in the municipalities of Cuernavaca, Chilpancingo, Guadalajara and Culiacán. A survey directed at seniors was used to explore the dependent variable (self-reporting of abuse) as well as the associated variables: sociodemographic characteristics, state of physical and mental health, social and family support and utilization of health services. For the statistical analysis non-parametric tests were used as well as a multivariate logistic regression model. Results: In this sample of seniors, $23 \%$ of the women and $9 \%$ of the men reported having been abused as a consequence of family problems. The factors included in this study that are related to abuse of seniors are: female gender $(O R=3.73 ; C I=1.76-4.24)$, age of 60 to 69 $(O R=1.61 ; C I=1.06-2.42)$, being divorced or separated $(O R=2.28 ; C I=1.32-3.96)$, suffering from a higher number of diseases $(O R=1.07 ; C I=1.01-1.17)$, presenting evident, observable injuries, $(O R=2.89 ; C I=1.01-3.58)$, presenting depressive symptoms $(O R=1.68 ; C I=1.04-2.72)$, and not having adequate family support $(O R=2.91 ; C I=1.86-4.55)$. Conclusion: Health systems face an important challenge in abuse of seniors which is due, on one hand, to the rapid increase of this population, and on the other, a lack of material and specialized human resources. Abuse towards seniors can be exacerbated by the increase in social violence and changes in family structure and traditional gender roles. Confronting this problem adequately requires providing comprehensive care tailored to the needs of seniors, the participation of all sectors of society, strengthening current knowledge, sensitizing society to this issue, and developing structures that permit the timely and efficient provision of health services.

Keywords: abuse in seniors citizens, marginalized settlements, Mexico.

\section{INTRODUCCIÓN}

La Comisión de Determinantes Sociales de la Salud de la OMS ha reportado que la exclusión social hacia los ancianos es una práctica que se observa en casi todos los países del mundo, destacando la invisibilidad social de este grupo, la distribución inequitativa de bienes y servicios, y el maltrato sobretodo en aquellos ancianos que viven en pobreza ${ }^{1}$.

En la Declaración de Toronto para la Prevención Global del Maltrato de las Personas Mayores ${ }^{2}$ se definió al maltrato como "la acción única o repetida, o la falta de respuesta apropiada, que ocurre dentro de cualquier relación donde exista una expectativa de confianza y la cual produzca daño o angustia a una persona anciana"' En cualquiera de los escenarios, es necesario destacar que el anciano maltratado es víctima de sufrimientos innecesarios, de lesiones o dolor, pérdida o violación de sus derechos humanos y deterioro generalizado de su calidad de vida. El maltrato es una manifestación de la violencia social y familiar que afecta a miles de individuos en el mundo ${ }^{3}$.
La violencia es un importante problema de salud pública en todo el mundo por las graves consecuencias que tiene para la salud y para el desarrollo psicológico y social de los individuos, las familias, las comunidades, los países, asimismo por las consecuencias en los servicios de salud al afectar los costos por los servicios ${ }^{4}$. Por ejemplo, en 1997 el costo de los gastos de asistencia sanitaria derivados de la violencia fue de $1.3 \%$ del Producto Interno Bruto en México ${ }^{5,6}$.

Entre los principales obstáculos del sistema de salud para dimensionar y enfrentar el problema del maltrato se encuentra el desconocimiento de la verdadera magnitud del problema debido, en gran parte, a la ausencia de denuncias de este tipo de delitos ${ }^{2}$. Algunos estudios realizados en el ámbito internacional ${ }^{7,8}$ indican que entre los factores que agravan el riesgo de maltrato, descuido y explotación, se encuentran el deficiente estado de salud de los ancianos, la depresión y la edad avanzada; así como vivir en condiciones de pobreza, carecer de apoyo familiar, el aislamiento social y la complicada relación de interdependencia y vinculación emocional de la persona maltratada y su agresor' ${ }^{9}$. Otros factores 
agravantes son el arraigo cultural de las premisas sociales que "normalizan" la violencia familiar, la percepción de que este problema es de índole privada y las reacciones de vergüenza, temor o desprestigio que conlleva hablar de este problema en público, los cuales tienen un papel subyacente importante. Cabe hacer mención que la mayor parte de los casos de maltrato en el anciano tiene lugar en el seno familiar, ${ }^{9}$.

Las escasas encuestas de base poblacional realizadas en otros países, indican que entre el $4 \%$ y el $6 \%$ de la población de edad avanzada sufre algún tipo de maltrato en el hogar ${ }^{10,11,12}$ aunque se calcula que solamente uno de cada cinco casos se denuncia a las autoridades. Por otra parte, estudios realizados en Canadá, el Reino Unido, España, Estados Unidos ${ }^{13}$, Colombia ${ }^{14}$ y Chile $^{15}$, así como reportes de la $\mathrm{OMS}^{16}$, indican que el maltrato en el adulto mayor es un problema prevalente y creciente en todo el mundo. Por ejemplo, en una reciente revisión sistemática de la literatura internacional sobre el tema ${ }^{17}$ se informa que el rango de maltrato (psicológico, verbal, físico y negligencia) reportado en estudios poblacionales es muy amplio, de $3.2 \%$ a $27.5 \%$, lo cual posiblemente refleja variaciones culturales de abuso, así como problemas conceptuales y metodológicos en torno a la medición de abuso, maltrato y negligencia. Sin embargo, los autores concluyen que estas cifras subestiman la tasa real de conductas violentas hacia los ancianos, ya que este grupo poblacional generalmente es renuente a hacer este tipo de reportes. Por otro lado, en el mismo documento se estima que los miembros de la familia que cuidan a los ancianos, reportaron haberlos maltratado con rangos de entre $5 \%$ a $55 \%$. Nuevamente, los autores proponen que este es un subregistro de conductas violentas.

En México la encuesta sobre Violencia Intrafamiliar de $1999^{18}$, registró que el $18.6 \%$ de la población de 60 años y más vivía en hogares donde se presentaban actos violentos. Por su parte el Instituto Nacional de las Personas Adultas Mayores, en el año 2001 reportó que en ese año, se presentaron 9 mil denuncias por violación a los derechos humanos en ancianos y para el año 2002 esta cifra ascendió a $18 \mathrm{mil}^{5}$.

El sector salud está frecuentemente en la primera línea de contacto con las víctimas de la violencia, además de que puede ayudar a las personas expuestas a riesgos ${ }^{3}$. Con el fin de proporcionar un posible perfil del adulto mayor maltratado en México para la detección y desarrollo de programas de prevención e intervención, así como para el diseño e implementación de políticas en el sistema de cuidados a la salud, es importante analizar las características de la población añosa a partir del auto-reporte de maltrato. La hipótesis que guió este trabajo fue que las características sociodemográficas, las condiciones y el deterioro físico y mental del adulto mayor, así como la no utilización de servicios de salud se encontraban relacionadas con la experiencia de maltrato en adultos mayores.

El objetivo de la presente investigación fue identificar algunos factores sociodemográficos (sexo, edad, estado civil e ingresos económicos); de salud (multimorbilidad, lesiones, sintomatología depresiva y consumo de alcohol); de apoyo social (familiar y de personas que no vivan con el adulto mayor); $y$, de servicios de salud (uso de los servicios de urgencias y de instituciones) que se asocian con el maltrato -a partir del auto-reporte- en adultos mayores que vivían en colonias urbano marginales en cuatro estados de la República Mexicana.

\section{MATERIAL Y MÉTODO}

Se realizó un estudio transversal en la República Mexicana, entre junio del 2004 y agosto del 2005, con adultos mayores (60 años y más) que residían en colonias marginadas de los municipios de Cuernavaca, Chilpancingo, Guadalajara y Culiacán.

El muestreo fue no probabilístico y en cada uno de los cuatro municipios se fijó una cuota de 100 hombres y 100 mujeres, a los cuales se les administró un cuestionario diseñado especialmente para este estudio. Los participantes fueron reclutados usando listados de los dirigentes de las colonias, centros de salud, iglesias y referencias comunales. Las colonias en donde se trabajó habían sido identificadas por las autoridades municipales respectivas y por el 
Tabla 1. Características de adultos mayores de acuerdo a reporte de maltrato.

\begin{tabular}{|c|c|c|c|c|c|}
\hline \multirow[t]{2}{*}{ VARIABLES } & \multicolumn{2}{|c|}{ NO MALTRATADOS } & \multicolumn{2}{|c|}{ MALTRATADOS } & \multirow[t]{2}{*}{$\mathbf{P}$} \\
\hline & $\mathrm{n}=669$ & $(\%)$ & $\mathrm{n}=130$ & $(\%)$ & \\
\hline $\begin{array}{l}\text { Sexo } \\
\text { Hombre } \\
\text { Mujer }\end{array}$ & $\begin{array}{l}357 \\
312\end{array}$ & $\begin{array}{l}53,36 \\
46,64\end{array}$ & $\begin{array}{l}37 \\
93\end{array}$ & $\begin{array}{l}28,46 \\
71,54\end{array}$ & 0,000 \\
\hline $\begin{array}{c}\text { Edad } \\
60-69 \\
70-79 \\
>80\end{array}$ & $\begin{array}{r}362 \\
214 \\
93\end{array}$ & $\begin{array}{l}54,11 \\
32,00 \\
14,00\end{array}$ & $\begin{array}{r}85 \\
39 \\
6\end{array}$ & $\begin{array}{r}65,00 \\
30,00 \\
5,00\end{array}$ & 0,006 \\
\hline Años de escuela cursados * & Media 1,85 & DE 5,48 & Media 18,9 & DE 2,97 & 0,548 \\
\hline $\begin{array}{l}\text { Estado civil } \\
\text { Tiene compañero (a) } \\
\text { No tiene compañero (a) }\end{array}$ & $\begin{array}{l}384 \\
285\end{array}$ & $\begin{array}{l}57,40 \\
42,60\end{array}$ & $\begin{array}{l}63 \\
67\end{array}$ & $\begin{array}{l}48,46 \\
51,54\end{array}$ & 0,007 \\
\hline $\begin{array}{c}\text { Hijos } \\
\text { Sí }\end{array}$ & 629 & 94,02 & 124 & 95,38 & 0,541 \\
\hline $\begin{array}{l}\text { Con quien vive } \\
\text { Esposa únicamente } \\
\text { Hijos únicamente } \\
\text { Esposa e Hijos } \\
\text { Familia y otros } \\
\text { Solo }\end{array}$ & $\begin{array}{r}140 \\
146 \\
135 \\
169 \\
79\end{array}$ & $\begin{array}{l}20,93 \\
21,82 \\
20,18 \\
25,26 \\
11,81\end{array}$ & $\begin{array}{l}19 \\
30 \\
25 \\
37 \\
19\end{array}$ & $\begin{array}{l}14,62 \\
23,08 \\
19,23 \\
23,46 \\
14,62\end{array}$ & 0,298 \\
\hline $\begin{array}{l}\text { Trabaja } \\
\text { Sí }\end{array}$ & 263 & 39,31 & 49 & 37,69 & 0,797 \\
\hline $\begin{array}{l}\text { Recibe apoyo económico } \\
\text { No }\end{array}$ & 370 & 55,31 & 70 & 53,85 & 0,759 \\
\hline Núm. enfermedades* & Media 3,82 & DE 2,64 & Media 4,73 & DE 2,81 & 0,000 \\
\hline Núm. lesiones* & Media 0,42 & DE 0,63 & Media 0,63 & DE 0,80 & 0,000 \\
\hline $\begin{array}{l}\text { Lesiones evidentes } \\
\text { a la observación } \\
\text { Sí }\end{array}$ & 51 & 7,62 & 20 & 15,38 & 0,000 \\
\hline $\begin{array}{l}\text { Presenta sintomatología } \\
\text { depresiva } \\
\text { Sí }\end{array}$ & 99 & 14,80 & 44 & 33,85 & 0,000 \\
\hline $\begin{array}{l}\text { Consumo de alcohol } \\
\text { No consumo } \\
1-2 \text { copas por ocasión } \\
\geq 3 \text { copas por ocasión }\end{array}$ & $\begin{array}{r}447 \\
163 \\
59\end{array}$ & $\begin{array}{r}66,82 \\
24,36 \\
8,82\end{array}$ & $\begin{array}{r}96 \\
27 \\
7\end{array}$ & $\begin{array}{r}73,85 \\
20,77 \\
5,38 \\
\end{array}$ & 0,230 \\
\hline $\begin{array}{l}\text { Apoyo familiar } \\
\text { Inadecuado }\end{array}$ & 110 & 16,44 & 53 & 40,77 & 0,000 \\
\hline $\begin{array}{l}\text { Apoyo instrumental social } \\
\text { Adecuado }\end{array}$ & 320 & 47,83 & 82 & 63,08 & 0,001 \\
\hline $\begin{array}{l}\text { Apoyo emocional social } \\
\text { Adecuado }\end{array}$ & 196 & 29,30 & 63 & 48,46 & 0,000 \\
\hline $\begin{array}{l}\text { Cuidador de otro } \\
\text { Sí } \\
\end{array}$ & 217 & 32,44 & 58 & 44,62 & 0,007 \\
\hline $\mathrm{N}^{0}$. hrs. al día solo* & Media 5,08 & DE 6,79 & Media 6,97 & DE 7,43 & 0,001 \\
\hline $\begin{array}{l}\text { Acudió a servicio de } \\
\text { urgencias } \\
\text { Sí }\end{array}$ & 148 & 22,12 & 34 & 26,15 & 0,553 \\
\hline $\begin{array}{l}\text { Derechohabiente } \\
\text { No } \\
\end{array}$ & 376 & 56,20 & 75 & 57,69 & 0,551 \\
\hline $\begin{array}{l}\text { Tipo de SS al que acude } \\
\text { IMSS } \\
\text { Centro de Salud } \\
\text { Médico Particular } \\
\text { Diversos servicios } \dagger\end{array}$ & $\begin{array}{l}177 \\
100 \\
165 \\
227\end{array}$ & $\begin{array}{l}26,46 \\
14,95 \\
24,66 \\
33,93\end{array}$ & $\begin{array}{l}31 \\
23 \\
27 \\
49\end{array}$ & $\begin{array}{l}23,85 \\
17,69 \\
20,77 \\
37,69\end{array}$ & 0,856 \\
\hline
\end{tabular}

* Variables continuas, Prueba de Man Withney.

$\dagger \quad$ IMSS, centros de salud, médico particular, farmacias, dispensarios, entre otros. 93 Fuente: Encuesta efectuada de junio del 2004 - agosto 2005 para el proyecto de investigación "Envejecimiento, 
Consejo Estatal de Población (Coespo) como de "muy alta" y "alta" marginación, con base en los criterios de marginación establecidos por Consejo Nacional de Población (Conapo) ${ }^{19}$. Se excluyeron a los ancianos que vivieran en casas de reposo o asilos, presentaran deterioro cognitivo de leve a severo y/o problemas de hipoacusia y trastornos del lenguaje, para lo cual, antes de la administración de la encuesta se aplicó una versión breve -modificada por las investigadoras- del mini-mental-test de Folstein ${ }^{20}$.

El protocolo de investigación fue evaluado y aprobado por las Comisiones de Investigación, Ética y Bioseguridad del Instituto Nacional de Salud Pública de México. Además, durante el contacto inicial con los participantes, se les explicó el objetivo del estudio, asegurándoles confidencialidad y anonimato en sus respuestas; también se les pidió su consentimiento informado por escrito.

El cuestionario, previamente probado, fue administrado por encuestadores con una escolaridad mínima de licenciatura, quienes fueron capacitados en un curso de $40 \mathrm{hrs}$. sobre el manejo y aplicación del instrumento, el cual incorporó un total de 11 secciones. Para el propósito de este trabajo las secciones que se incluyeron en el análisis fueron: 1) Datos sociodemográficos; se incluyó el sexo, edad (60-69, 70-79, >80 años), educación, estado civil, presencia de hijos, con quien vive, actividad laboral e ingresos de diversas fuentes. 2) La situación actual de salud se evaluó preguntando el tipo y número de enfermedades que habían padecido en el último año y si en el mismo período habían tenido alguna lesión (herida, contusión, quemadura, luxación, fractura, otros). Las lesiones fueron caracterizadas por tipo y región anatómica afectada y lugar en donde ocurrió la lesión, así mismo fueron clasificadas por causa externa y codificadas de acuerdo a la Clasificación Internacional de Enfermedades (CIE-10). Por otra parte, si el anciano mostraba lesiones evidentes a la observación del encuestador, éstas fueron anotadas en un formato anexo al cuestionario, al momento de la entrevista. 3) La sintomatología depresiva se evaluó a través de la Escala de Depresión CES-D (Radloff, 1977) ${ }^{21}$, que en esta muestra obtuvo un Alpha de
0.86 . Se tomó como punto de corte la media más una desviación estándar, de acuerdo a estudios previos $^{22}$, para considerar un posible caso de depresión. Se midió también el uso de alcohol a través de frecuencia, cantidad y tipo de bebida. 4) Se evaluaron dos tipos de apoyo el social y el familiar. Para el primero se preguntó si contaba con alguna persona, que no vivieran con ellos, que les brindara apoyo instrumental (facilitando las tareas cotidianas) y emocional (con quien hablar, que les generara sentimientos de bienestar afectivo). Se preguntó también el número de horas al día que estaba solo y si el anciano era cuidador de otra persona. El apoyo familiar se evaluó mediante una escala de 13 reactivos $^{23}$ -ejemplo: ¿Qué tanto se ocupan realmente de usted sus familiares?- que se respondían en una escala Likert de cuatro puntos (siempre/mucho $=1$; a nunca $/$ nada $=4$ y se tomó como punto de corte la media más una desviación estándar para considerar que el apoyo familiar no era "adecuado". La escala de apoyo familiar obtuvo un Alpha de 0.88. 5). Se indagó si habían acudido a algún servicio de urgencias el último año, si eran derechohabientes y el tipo de servicios al que acudían cuando necesitaban atención médica. 6) Por último, se preguntó a los ancianos "alguna vez ha sido maltratado (a) como consecuencia de algún conflicto familiar". Esta constituyó la variable dependiente, el ponderado final quedó (0) para aquellos que no reportaron haber sido maltratados y (1) para los que si reportaron haber sido maltratados.

\section{Análisis de datos}

Se realizó un análisis exploratorio de cada una de las variables con la finalidad de evaluar la calidad de la información y describir a la población de estudio. Se calcularon las medidas de tendencia central y dispersión para las variables continuas. En el caso de las variables categóricas, se obtuvieron las frecuencias para cada categoría, así como el porcentaje correspondiente. En el análisis bivariado para explorar la relación entre maltratados y no maltratados, se utilizaron la prueba de Ji cuadrada y la prueba Man Withney, dada la distribución de la población. Igualmente se empleó la regresión logística binaria simple y 
se calcularon razones de momios (RM).

Se ajustó un modelo multivariado de regresión logística binaria con el propósito de obtener las mejores variables asociadas a las lesiones en los adultos mayores. La fuerza de asociación en el modelo fue expresado como razones de momios con intervalos de confianza al 95\% (IC 95\%), reportándose igualmente los valores de $\mathrm{p}$. En el modelo multivariado final, se incluyeron las variables que en el análisis bivariado tuvieron un valor de $<0.25$. Se realizó el análisis del factor de inflación de la varianza (VIF) con el propósito de evaluar la multicolinealidad entre las variables independientes. Se probaron interacciones y permanecieron en el modelo si su valor de $\mathrm{p}$ fue menor a 0.15 . La prueba de error de especificación (linktest) fue usada para probar el supuesto que asume que el logit de la variable de respuesta es una combinación lineal de las variables independientes. El ajuste del modelo se realizó con la prueba de bondad de ajuste usando un punto de corte de $\mathrm{p}>0.10$ para considerar un ajuste adecuado ${ }^{24}$.

Para el procesamiento de la información se utilizó el paquete estadístico Stata, versión 9.

\section{RESULTADOS}

La muestra estuvo conformada por 799 adultos mayores, 394 fueron hombres (49\%) y 405 mujeres $(51 \%)$, con un promedio de educación escolar de $1.8+5.4$. El $44 \%$ no tenía pareja; $94 \%$ tenía hijos; el 37\% vivía con otros familiares, amigos o solo; $39 \%$ trabajaba en varios oficios (albañil, pintor, jardinero, y comercio informal, entre otros) y $55 \%$ indicó no recibir ningún tipo de apoyo económico fuera de sus escasos ingresos por trabajo remunerado. Asimismo, el 23\% de las mujeres y el $9 \%$ de los hombres encuestados reportaron haber sido víctimas de maltrato.

En la Tabla 1 se describen las características generales de los participantes, sociodemográficas, salud, apoyo social y servicios de salud comparando al $16.3 \%(n=130)$ de la muestra que fueron los ancianos que indicaron haber sido maltratados, con el resto de los participantes $(83.7 \%)(\mathrm{n}=669)$ que no reportaron maltrato, así como los resultados observados en las pruebas de Ji2 o Man Withney según la variable.

La Tabla 1 sugiere que entre los maltratados existe una mayor proporción de mujeres; un mayor porcentaje de adultos entre 60 y 69 años $(\mathrm{p}<0.05)$; una mayor proporción $(\mathrm{p}<0.05) \mathrm{de}$ personas que no tenía compañero (solteros, divorciados o viudos), así como de adultos mayores que vivían con sus hijos $(\mathrm{p}>0.05)$. Una proporción menor de los que trabajaban y tenían apoyo económico $(\mathrm{p}>0.05)$. La media en número de enfermedades y número de lesiones fue mayor en los que reportaron maltrato y fue más alta la proporción de ancianos maltratados que presentaban lesiones evidentes a la observación, sintomatología depresiva y apoyo familiar inadecuado. Sin embargo, contaban con mayor apoyo social tanto instrumental como emocional de personas que no vivían con ellos $(\mathrm{p}<0.05)$. En este grupo también fue mayor el porcentaje de los que reportaron ser cuidadores de otros $(\mathrm{p}<0.05)$.

$\mathrm{Al}$ analizar los datos por sexo al interior del grupo de los maltratados encontramos entre las mujeres una proporción significativamente más alta $(\mathrm{p}<0.05)$ de mujeres sin compañero (divorciada, separada o viuda), que reportan un mayor número de lesiones, que son cuidadoras de otros y que cuentan con apoyo instrumental. En los hombres la única variable con significancia estadística fue presentar lesiones evidentes a la observación.

En cuanto al uso de servicios de salud, aunque no se encontró significancia estadística, los datos sugieren que una mayor proporción de adultos mayores maltratados que sus contrapartes, acude tanto a los servicios de urgencia $(26.2 \%$ vs. $22.1 \%$ ) como a diversas fuentes de cuidados de salud (37.7\% vs $33.9 \%)$ como el IMSS, los centros de salud, médicos particulares, farmacias y dispensarios médicos.

Por otra parte, $61(47 \%)$ adultos mayores que señalaron haber sido maltratados reportaron haber tenido una o más lesiones el año previo a la encuesta. El total de lesiones reportadas fue de 83 que se distribuyeron de la siguiente manera: 44 personas reportaron una lesión; 12 reportaron dos lesiones; y sólo cinco manifestaron tener o haber tenido tres o más lesiones en el último año. 
La distribución por tipo de lesión fue: heridas 19 (23\%), contusiones $26(31 \%)$, quemaduras 10 $(12 \%)$, luxaciones $20(24 \%)$ y fracturas $8(10 \%)$. Las partes del cuerpo afectadas por las lesiones fueron: extremidades superiores e inferiores 67 $(80 \%)$, cabeza $9(11 \%)$ y cadera $7(9 \%)$. La mayor proporción de lesiones ocurrió en el hogar (63.94\%). Al codificar estas lesiones por causa externa quedaron: $12(20 \%)$ por fuerzas mecánicas inanimadas, $33(54 \%)$ por caídas, 4 (7\%) por fuego y sustancias calientes, 2 (3\%) por otro tipo de causa externa y $10(16 \%)$ por dos o más causas externas.

Es importante señalar que 71 participantes presentaron lesiones en partes visibles del cuerpo, las cuales fueron evidentes a la observación del encuestador. Sin embargo, 17 (24\%) no las reportaron. Del resto de ancianos que sí reportó haber tenido lesiones, el 38\% indicó que las lesiones habían sido ocasionadas tanto de manera accidental como intencional.

Finalmente, en la Tabla 2 se presenta el modelo multivariado en el cual se pueden observar los factores que se relacionaron con el maltrato como consecuencia de problemas familiares. Se estimó que el ser mujer incrementa en 2.73 los momios ( $\mathrm{IC}=1.76$ 4.24) de ser maltratada en relación con ser hombre. Los adultos mayores que tienen entre 60 a 69 años de edad tuvieron $61 \%$ más posibilidades de haber sufrido maltrato $(\mathrm{RM}=1.61 ; \mathrm{IC}=1.06-2.42)$ en relación con los otros grupos de edad. Se observó que la posibilidad de ser víctima de maltrato aumenta 1.28 veces cuando no tienen compañero $(\mathrm{RM}=2.28 ; \mathrm{IC}=1.32-3.96)$. Se estimó que conforme aumenta el número de enfermedades $(\mathrm{RM}=1.07 ; \mathrm{IC}=1.01-1.17)$ la posibilidad de ser maltratado aumenta. El presentar lesiones evidentes a la observación en partes visibles del cuerpo aumenta 1.89 los momios de haber sido maltratado $(\mathrm{IC}=1.01-3.58)$. Finalmente, la presencia de sintomatología depresiva y apoyo familiar inadecuado incrementan los momios ( $\mathrm{R} \mathrm{M}=1.68 ; \mathrm{I} \mathrm{C}=1.04-2.72) \quad \mathrm{y}$ $(\mathrm{RM}=2.91 ; \mathrm{IC}=1.86-4.55)$, respectivamente, en quienes reportaron haber sido maltratados.

\section{DISCUSIÓN}

Los resultados de este trabajo muestran una prevalencia importante de maltrato hacia los adultos mayores, sobre todo mujeres, ya que el $23 \%$ de las ancianas y el $9 \%$ de los hombres encuestados reportaron haber sido víctimas de maltrato asociado generalmente a problemas familiares. Estos hallazgos son consistentes con aquellos reportados en la Encuesta sobre Violencia Intrafamiliar llevada a cabo en México hace 10 años, en la cual se reportó que el $18.6 \%$ de la población de 60 años y más vivía en hogares donde se presentaban actos violentos.

Entre los factores que en estudio se encontraron asociados con el maltrato autoreportado por los adultos mayores que participaron en este estudio se encuentran: sexo femenino, edad de 60 a 69 años, no tener compañero, reportar un mayor número de enfermedades, presentar lesiones evidentes a la observación, tener sintomatología depresiva y apoyo familiar inadecuado, todo lo cual es consistente con estudios previos sobre el tema $8,15,17,25$.

Por otra parte, los roles sociales influenciados por los aspectos culturales, sobre todo en torno al género, se reflejan al ubicarse la mujer con una mayor vulnerabilidad en relación con el hombre, confirmando de acuerdo con otros estudios, que es mucho más frecuente observar el maltrato en la mujer ${ }^{26}$, en este estudio, el maltrato afectó a una mayor proporción de mujeres, que no tenía compañero y que además era cuidadora de otros. Las mujeres son las principales cuidadoras de otros en los grupos pobres $^{27}$, sobre todo de la vejez ${ }^{28}$. Esta actividad se suma a los diversos y múltiples roles sociales que simultáneamente ejercen las ancianas. El ser cuidador de otros ha comenzado a ser un área prioritaria en el campo de la salud, debido al envejecimiento, al aumento de las enfermedades crónicas en los panoramas demográficos y epidemiológicos ${ }^{29}$. Aun cuando el ser cuidadora de otros no fue significativo en el modelo final, esta actividad es considerada como un factor de riesgo para el maltrato, debido el estrés que esta práctica provoca en el cuidador ${ }^{4}$. 
Tabla 2. Análisis multivariado de regresión logística de adultos mayores que reportaron maltrato como consecuencia de problemas familiares.

\begin{tabular}{|c|c|c|}
\hline VARIABLES & $\begin{array}{l}\text { RM Ajustada } \\
\text { (IC } 95 \%)\end{array}$ & $\mathbf{P}$ \\
\hline $\begin{array}{l}\text { Sexo } \\
\text { Hombres } \\
\text { Mujeres }\end{array}$ & $\begin{array}{c}1^{*} \\
2,73(1,76-4,24)\end{array}$ & 0,000 \\
\hline $\begin{array}{l}\text { Edad } \\
\quad>70 \\
60-69\end{array}$ & $\begin{array}{c}1^{*} \\
1,61(1,06-2,42)\end{array}$ & 0,025 \\
\hline $\begin{array}{l}\text { Estado civil } \\
\text { Tener } \\
\text { compañero } \\
\text { No tener } \\
\text { compañero }\end{array}$ & $\begin{array}{c}1 * \\
2,28(1,32-3,96)\end{array}$ & 0,003 \\
\hline $\begin{array}{l}\mathbf{N}^{\mathbf{0}} \\
\text { Enfermedades }\end{array}$ & $1,07(1,01-1,17)$ & 0,045 \\
\hline 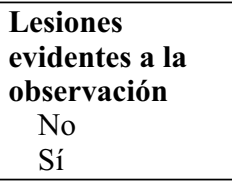 & $\begin{array}{c}1^{*} \\
1,89(1,01-3,58) \\
\end{array}$ & 0,049 \\
\hline $\begin{array}{l}\text { Presentar } \\
\text { sintomatología } \\
\text { depresiva } \\
\text { No } \\
\text { Sí }\end{array}$ & $\begin{array}{c}1^{*} \\
1,68(1,04-2,72)\end{array}$ & 0,032 \\
\hline $\begin{array}{c}\text { Apoyo Familiar } \\
\text { Adecuado } \\
\text { Inadecuado }\end{array}$ & $\begin{array}{c}1^{*} \\
2,91(1,86-4,55)\end{array}$ & 0,000 \\
\hline
\end{tabular}

Fuente: Encuesta efectuada de junio del 2004 - agosto 2005 para el proyecto de investigación "Envejecimiento, Pobreza y salud en población urbana".

* Referencia

- Factor de Inflación de varianza $(\mathrm{VIF})=1,06$, tolerancia 0,9

- Prueba de Bondad de Ajuste 1fit, group (10): number of observations $=799$; Hosmer-Lemeshow chi2(8) = 2,86; Prob $>$ chi $2=0,9430$.

- Prueba de error de especificación (linktest) hat $\mathrm{p}=0,003$; hatsq $\mathrm{p}=0,301$.

En este estudio se observó que una cuarta parte de los ancianos que presentaba lesiones evidentes no las reportó y el 38\% indicó que fueron ocasionadas tanto de manera intencional como accidental. Se ha informado que en la mayoría de los casos las víctimas no perciben la violencia como una violación o abuso. Entre las razones para no denunciar están que la sociedad minimiza, tolera o promueve esos actos; que en algunos casos son amenazadas por el agresor; que desconocen los mecanismos de denuncia correspondientes o que lo reconocen como parte de una cultura. También hay miedo a la reacción de los funcionarios, a la vergüenza y al temor a las consecuencias que pueda tener la denuncia. En ocasiones al denunciar los abusos los adultos mayores se enfrentan al manejo inadecuado del reporte por la falta de capacitación de los servidores públicos ${ }^{5,30}$. Entre los principales obstáculos que enfrenta el sistema de salud para conocer la dimensión del problema de maltrato en el adulto mayor se encuentran la ocultación de la información tanto de la víctima como de las personas involucradas en los actos de maltrato.

Estudios anteriore ${ }^{17}$ sugieren que la mejor forma de detectar el maltrato no es la observación ni búsqueda de lesiones, sino preguntar de manera rutinaria a los ancianos y sus cuidadores sobre conductas violentas y posible maltrato verbal, psicológico y físico por parte de sus familiares y su red social. Esta aproximación directa para identificar el problema es mucho más sensible que la observación, aunque en casos de abuso severo, el miedo que puede producir el reporte verbal del maltrato, promueve el silencio y el ocultamiento del problema.

Que el paciente anciano esté en contacto con los servicios de salud constituye una oportunidad para que los prestadores de salud puedan identificar el maltrato en las personas de edad, así como para prevenir y tratar la violencia contra ellos. No obstante, para poder desempeñar esta función necesitan una formación y una orientación específicas que aumenten su grado de conciencia del problema ${ }^{31}$, así como contar con guías, protocolos y un sistema de rastreo. Entre las recomendaciones que señala la OPS para identificar el problema de maltrato en un adulto mayor, se encuentra "realizar una evaluación geriátrica integral del paciente, con inclusión de los aspectos médicos, funcionales, cognoscitivos y sociales"4. Sin embargo, se carece de infraestructura y de especialistas para proporcionar servicios de este tipo ${ }^{5}$.

Entre las limitaciones de este estudio se encuentra el diseño transversal de la investigación, lo cual no permite establecer causalidad entre las 
variables dependiente e independientes. Por ejemplo, las lesiones y la depresión han sido considerados factores de riesgo para el maltrato ${ }^{6}$, pero también pueden ser consecuencia tanto de maltrato ${ }^{3}$ como de enfermedades crónicas o de multimorbilidad en los ancianos ${ }^{32}$. Por otro lado, aún cuando los resultados de la presente investigación no pueden ser extrapolados a la población en general, los hallazgos de este estudio contribuyen a ampliar el conocimiento sobre las características de la población adulta mayor en México que puede potencialmente sufrir de maltrato. La información presentada puede servir como una herramienta útil para planear servicios de salud, generar programas de prevención y promoción y para el desarrollo de investigaciones futuras sobre los factores de riesgo asociados al maltrato en el adulto mayor en México. Finalmente, a pesar de que los indicadores subjetivos han sido cuestionados por su dependencia cultural, educativa y factores étnicos, el auto-reporte en salud ha representado una medición costo-efectiva en estudios epidemiológicos y se ha empleado en varios aspectos relacionados a la salud ${ }^{33}$. Los indicadores subjetivos han sido reconocidos como indicadores útiles entre grupos de adultos mayores, entre otras razones porque pueden ser aplicados fácilmente mediante encuestas de una sola pregunta y permiten la colección de una gran diversidad de datos ${ }^{34}$.

El sistema de salud y la sociedad se enfrentan a un reto importante, aunque de magnitud desconocida: el maltrato en los adultos mayores. Es importante contar con un sistema de vigilancia preciso que dé cuenta precisa de la magnitud del problema, ya que no todo maltrato ocasiona lesiones graves como para requerir atención médica. La naturaleza del maltrato hacia los ancianos y su invisibilidad constituyen un doble desafío para los sistemas de salud, y demanda acciones y políticas focalizadas a la resolución del problema. Por ejemplo, el extender servicios básicos de salud para incorporar la identificación del maltrato requiere primero la concientización del sector salud en torno al envejecimiento poblacional global, así como la capacitación de recursos humanos especializados. Finalmente, resulta evidente que la colaboración de entidades gubernamentales junto con el sector privado y las organizaciones de la sociedad civil deba ser incentivada, así como la participación comunitaria, la cual puede ser clave para el diseño e implementación de programas exitosos que no sólo reviertan la exclusión social que han experimentado los ancianos sino que den voz a los más vulnerables.

Financiado por El Fondo Sectorial SALUD del Consejo Nacional de Ciencia y Tecnología de México (CONACYT) "Envejecimiento, Pobreza y Salud en Población Urbana" (Proyecto 2002-CO1-7419).

\section{REFERENCIAS}

1. POPEY J, ESCORCEL S, HERNANDEZ M, JOHNSTON H, MATHIESON J, RISPEL L. Understanding and Tackling Social Exclusion. Final Report to the WHO Commission on Social Determinants of Health From the Social Exclusion Knowledge Network. OMS. Febrero 2008.

2. INTERNATIONAL SOCIETY FOR EQUITY IN HEALTH. Toronto Declaration on Equity in Health. Rev Panam Salud Pública/Pan Am J Public Health, $2002 ; 12(6)$

3. ORGANIZACIÓN DE LAS NACIONES UNIDAS. El maltrato de las personas de edad: reconocer y responder al maltrato de las personas de edad en un contexto mundial. Comisión de Desarrollo Social constituida en comité preparatorio de la Segunda Asamblea Mundial sobre el Envejecimiento. 2002. E/CN.5/2002/PC/2

4. ORGANIZACIÓN MUNDIAL DE LA SALUD. $49^{\mathrm{a}}$. Asamblea Mundial de la Salud. Resolución. 1996

5. ORGANIZACIÓN DE LAS NACIONES UNIDAS. Diagnóstico sobre los derechos humanos en México. México: Mundi-prensa / Oficina del Alto Comisionado de las Naciones Unidas para los Derechos Humanos. 2003.

6. ORGANIZACIÓN PANAMERICANA DE LA SALUD. Informe mundial sobre la violencia y la salud. Washington: OPS. 2003.

7. ORGANIZACIÓN DE LAS NACIONES UNIDAS. Abuse of older persons: recognizing and responding to abuse of older persons in a global context. Commission for Social Development acting as the preparatory committee for the Second World Assembly on Ageing 2002 E/CN.5/2002/PC/2

8. Programa de actividades preventivas y de promoción a la salud. Malos tratos a los ancianos. PAPPS. Madrid. 2002.

9. CORSI J. Violencia familiar. Una mirada 
interdisciplinaria sobre un grave problema social. Argentina. Paidos 2001.

10. BRUBAKER TH. An overview of family relationships in later life. Newbury Park, CA: Sage 1983.

11. PILLERMER KA, FINKELHOR D The prevalence of elder abuse: a random sample survey. The Gerontologist 1988:28(1):51-57.

12. PODNIEKS E, National survey on abuse of the elderly in Canada. Journal of Elder Abuse \& Neglect 1992; 4(3): 5-58

13. ORGANIZACIÓN DE LAS NACIONES UNIDAS. Asamblea Mundial sobre el envejecimiento. 2003.

14. MÁRQUEZ H. Abuso y maltrato. Red Latinoamericana de Gerontología. Colombia. 2001.

15. MARIN PP. Abuso o maltrato en adulto mayor. Boletín de la Escuela de Medicina. 2000;29:1

16. ORGANIZACIÓN MUNDIAL DE LA SALUD. Abuso y maltrato a las personas mayores: hacia una respuesta integral, Guatemala: OPS 2003.

17. COOPER C, SELWOOD A, LIVINGSTONE G. The prevalence of elder abuse and neglect: a systematic review. Age and Aging 2008; 37 (2): 151-160.

18. INSTITUTO NACIONAL DE ESTADÍSTICA Y GEOGRAFÍA DE MÉXICO. Estadísticas a propósito del día internacional de las personas de edad. México. INEGI 2003.

19. CONSEJO NACIONAL DE POBLACIÓN DE MÉXICO. Estadísticas índice y grado de marginación por localidad. México. CONAPO 2000.

20. FOLSTEIN MF, FOLSTEIN SE, MCHUGH PR. 'MiniMental State': a practical method for grading the cognitive state of patients for the clinician. Journal of Psychiatric Research 1975;12: 189-198.

21. RADLOFF, LS The CES-D Scale: A self-report depression scale for research in the general population. Applied Psychological Measurement, 1977; 1(3): 385401.

22. SALGADO DE SNYDER VN, MALDONADO M. Características psicométricas de la escala de depresión Centro de Estudios Epidemiológicos en mujeres mexicanas adultas de áreas rurales. Salud Pública de Mex. 1994;36: 200-209.

23. SALGADO DE SNYDER VN Envejecimiento, género y pobreza en México rural. En V.N. Salgado de Snyder y R. Wong (Eds). Envejeciendo en la pobreza: género, salud y calidad de vida. México: Instituto Nacional de Salud Pública. 2003 p. 37-56.
24. HOSMER DW, LEMESHOW S. Applied logistic regression. New York: Jonh Wiley \& Sons Interscience Publication 2000;2.

25. ORGANIZACIÓN MUNDIAL DE LA SALUD. Declaración de Hong Kong sobre el maltrato de los ancianos. 1989.

26. ORGANIZACIÓN MUNDIAL DE LA SALUD /ORGANIZACIÓN PANAMERICANA DE LA SALUD. Violencia contra la mujer: un tema de salud prioritario. Washington: Unidad de Salud de la Mujer 1998.

27. RANDEL J, GERMAN T, EWING D. HELPAGE INTERNATIONAL The ageing \& development report: independence and the world's older people. London, England: Earthscan Publications 1999.

28. SECRETARÍA DE SALUD DE MÉXICO. Programa Nacional de Salud 2001-2006. SSA: México 2001.

29. ROBLES SL. Género, pobreza y cuidado: la experiencia de mujeres cuidadoras pobres urbanas. En V.N. Salgado de Snyder y R. Wong (Eds). Envejeciendo en la pobreza: género, salud y calidad de vida. México: Instituto Nacional de Salud Pública. 2003 p.123-154.

30. SECRETARÍA DE SALUD DE MÉXICO. Violencia contra las mujeres. Un reto para la salud pública en México. Informe Ejecutivo de la Encuesta Nacional de Violencia contra las Mujeres. México: Instituto Nacional de Salud Pública. 2003.

31. DECALMER P, GLENDENNING F. El maltrato a las personas mayores. Argentina. Paidos. 1993.

32. ORGANIZACIÓN MUNDIAL DE LA SALUD Problemas de salud mental relacionados con el envejecimiento. Comunicados para la Salud 3. Washington: OPS 1992.

33. AVILA-BURGOS L, RAMÍREZ-VALVERDE G, MARTÍNEZ-DAMIAN MA, CRUZ-VALDEZ A, SANTIAGO-CRUZ MJ, MEDINA-SOLIS CE. Socioeconomic determinants of inequality and selfreportes morbidity among adolescents in a developing country. Saudi Med J 2005;26(10): 447 453.

34. WONG R, PELAEZ M, PALLONI A. Autoinforme de salud general en adultos mayores de América Latina y el Caribe: su utilidad como indicador. Rev Panam Salud Publica 2005;17:5-6.

Recepción: 17 agosto 2009 Aprobación: 9 noviembre 2009

Usted puede comentar éste y otros artículos publicados en la Revista Chilena de Salud Pública, enviando un correo electrónico a revistasp@med.uchile.cl 